\title{
Guest Editorial: Special Issue on THz Radiation Applied to Biophysical, Biological, and Biomedical Sciences
}

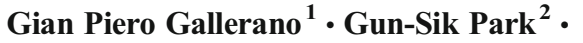 \\ Alfonsina Ramundo-Orlando ${ }^{3} \cdot$ Olga Zeni $^{4}$
}

Received: 21 June 2018 / Accepted: 24 June 2018 /Published online: 2 July 2018

(C) Springer Science+Business Media, LLC, part of Springer Nature 2018

In the 15 years following the pioneering "THz-BRIDGE Workshop 2002," several initiatives have been dedicated to the applications of $\mathrm{THz}$ radiation to biophysical, biological, and biomedical sciences. In 2010, a workshop series entitled "International THz-Bio Workshop" started in Korea with the support of the Ministry of Education, Science, and Technology and was extended to a worldwide geographical coverage in occasion of the 8th International THzBio Workshop held at ENEA-Frascati, Italy, from 4 to 6 October 2017.

The aim of the Workshop was to bring together scientists from different communities to discuss a broad range of scientific issues in the terahertz and adjacent parts of the infrared and microwave spectral regions. The current scenario and the open issues of different aspects were treated: spectroscopic measurements on biological systems of increasing complexity, mechanisms of interaction and effects induced by the electromagnetic field, safety issues, technological developments of terahertz active and passive instrumentations, and THz-Bio sensing and imaging. Selected invited papers presented at the Workshop, and a couple of other relevant papers, are collected in this Special Issue of the Journal of Infrared, Millimeter, and $\mathrm{THz}$ Waves aiming at providing the state-of-the-art of the applications of mm-waves and $\mathrm{THz}$ radiation in biophysical, biological, and biomedical fields.

Novel aspects of fundamental spectroscopic investigations are covered in the two papers, "Quantum electric dipole lattice: Water molecules confined to nanocavities in Beryl" and "Differences in hydration structure around hydrophobic and hydrophilic model peptides probed by THz time-domain spectroscopy," which address the peculiar role played by "bound water" in different confined systems. Specific spectroscopic investigations are then presented

Gian Piero Gallerano

gianpiero.gallerano@enea.it

ENEA, FSN - Fusion Physics Division, 00044 Frascati, Italy

2 Department of Physics and Astronomy, Seoul National University, Seoul, South Korea

3 CNR-IFT, 00133 Rome, Italy

4 CNR-IREA, 80124 Naples, Italy 
in the two papers, "Sharp absorption peaks in THz spectra of middle molecular weight pharmaceuticals" and "Albumin solutions comprehensive study in an extended THz frequency range," which systematically investigate the properties of pharmaceuticals and albumin solutions.

The paper "Electroporation-induced cell modifications detected with $\mathrm{THz}$ time-domain spectroscopy" bridges the gap between spectroscopic measurements and biological effects on living cells by presenting a technique that has a significant potential for biomedical applications. The status and perspectives of such applications are then discussed in the paper "Is there a biological basis for therapeutic applications of millimetre waves and THz waves?", which also investigates if published experimental studies, both in vivo and in vitro, provide evidence for "non-thermal" biological effects of mm-waves and $\mathrm{THz}$ radiation. In this paper, the quality of the studies is highlighted as a crucial issue that enables a more accurate analysis of the available data.

The potential of THz reflection imaging is discussed in the paper "Pixel statistical analysis of diabetic vs. non-diabetic foot-sole spectral terahertz reflection images," which demonstrates the importance of acquiring a large data set of comparative images to improve the reliability of $\mathrm{THz}$ imaging as a diagnostic tool.

Finally, four papers are dedicated to studies of mm-waves and THz-induced effects on skin tissue models and human fibroblasts, as well as on rabbit cornea and human oral cavity. The paper "Topology-based predictions of pathway dysregulation in human skin tissue models induced by intense Terahertz pulses" investigates the differential gene expression resulting after exposure of skin tissue models. The observed effects can lead to potential novel clinical applicability of intense $\mathrm{THz}$ pulses. The paper "Biological effects of $\mathrm{THz}$ and $\mathrm{MW}$ radiation on Human Fibroblasts" presents a comparison of results of exposure of human fibroblast to pulsed versus continuous wave $(\mathrm{CW})$ radiation. The papers "Ocular effects of exposure to 40, 75 , and $95 \mathrm{GHz}$ millimeter waves" and "Thermal impact on the human oral cavity exposed to radiation from biomedical devices operating in the $\mathrm{THz}$ frequency range" present a model of ocular damage induced by $\mathrm{CW}$ millimeter waves and a model to estimate electric field strengths and temperature elevations in the human oral cavity, respectively.

We sincerely thank the Editor-in-Chief of the journal, Prof. Martin Koch, for his support and encouragement in setting up this special issue. We also wish to thank the reviewers who have contributed in selecting the papers and improving the quality with their careful professional feedback. We hope that this special issue can provide a good reference for the present, stimulate further studies, and contribute to the development of this exciting field of research. 